\title{
Bio-active restorative materials with antibacterial effects: new dimension of innovation in restorative dentistry
}

\author{
Satoshi IMAZATO \\ Department of Restorative Dentistry and Endodontology, Osaka University Graduate School of Dentistry, 1-8 Yamadaoka, Suita, Osaka 565-0871, \\ Japan \\ Corresponding author, Satoshi IMAZATO; E-mail: imazato@dent.osaka-u.ac.jp
}

\begin{abstract}
Restorative materials in the new era should be "bio-active", and antibacterial effects are highlighted as one of the important properties. In order to achieve resin-based restoratives with antibacterial effects, an antibacterial monomer MDPB has been developed. The primer incorporating MDPB demonstrated cavity-disinfecting effects, and the world's first antibacterial adhesive system employing the MDPB-containing primer was successfully commercialized. MDPB is potentially applicable to various restoratives since immobilization of the antibacterial component by polymerization of MDPB enables no deterioration in mechanical properties of cured resins and exhibition of inhibitory effects against bacterial growth on their surfaces. For glass-ionomer cements used for atraumatic restorative treatment, the approach to provide antibacterial activity has been attempted by addition of chlorhexidine. Incorporation of $1 \%$ chlorhexidine diacetate was found to be optimal to give appropriate antibacterial and physical properties, being effective to reduce the bacteria in affected and infected dentin in vivo.
\end{abstract}

Key words: Restorative materials, antibacterial activity, MDPB

Received Sep 25, 2008: Accepted Dec 2, 2008

\section{INTRODUCTION}

Basic properties of dental materials for restorative treatment, such as mechanical, physical and bonding properties, have been greatly improved as a result of numerous investigations, and many of recent products on the market exhibit excellent/acceptable clinical performance. Such improvement of restorative materials has contributed to the recovery of ideal anatomical form and function with less removal of tooth structure, leading the way to aesthetic restorative treatments and minimal intervention dentistry. Accordingly, it is proposed that innovation of restorative materials in the new era could be directed toward a new dimension: development of materials with "bio-active functions" to provide therapeutic effects.

As one bio-active function proposed for restorative materials, antibacterial activity can be highlighted for the restorative treatment of caries. The ability to control bacteria would be advantageous to eliminate the risk of further demineralization and cavitation, since dental caries is an infectious disease and eradication of cariogenic bacteria is the important principle. We have been conducting research on development of various antibacterial resins by incorporation of a novel monomer 12-methacryloyloxydodecylpyridinium bromide (MDPB), which has antibacterial activity. The antibacterial adhesive incorporating this monomer has been successfully developed and is commercially available. In this review paper, our research work on MDPB, including its possible use for future treatment, is summarized. In addition, the development of novel glass-ionomer cements containing chlorhexidine for atraumatic restorative treatment (ART) is also addressed.

\section{ANTIBACTERIAL RESINS INCORPORATING MDPB}

\section{Antibacterial monomer $M D P B$}

In order to achieve resin-based restorative materials with antibacterial effects, we aimed to develop an antibacterial monomer that is a polymeirzable bactericide (Fig. 1). Several antimicrobials were tested for their possibility to be combined with a methacryloyl group, and MDPB was successfully synthesized from quaternary ammonium dodecylpyridinium (Fig. 2) ${ }^{1,2)}$.

When unpolymerized, antibacterial monomer acts as a free bactericide similar to conventional antimicrobials. MDPB, an analogue of cetylpyridinium chloride, which is a strong bactericide frequently used for oral rinses or dentifrices, was confirmed to have strong killing activity against various oral bacteria as proven by its minimum bactericidal concentration $(\mathrm{MBC})$ values (Table 1$)^{3,4)}$. In addition to facultative and obligate anaerobe detected in coronal lesions, MDPB is effective against bacterial species predominantly isolated from root caries, such as actinomyces and Candida albicans ${ }^{5}$. It was also found that MDPB can kill bacteria rapidly at 
concentrations beyond $\mathrm{MBC}^{6)}$.

MDPB is a polymerizable bactericide, and the antibacterial component in the molecule is immobilized after the resinous materials incorporating MDPB are cured (Fig. 3) ${ }^{1,2,7)}$. The component immobilized as a pendant does not leach out from resin matrix as they are connected by covalent bond $^{1,8)}$. Therefore, incorporation of MDPB has an advantage that physical properties of the restorative materials are kept stable for a long period under wet circumstances ${ }^{1,9)}$.

Antibacterial adhesive system containing $M D P B$ The experimental antibacterial dentin primer was prepared by incorporating MDPB into the primer of a commercial self-etching system (Liner Bond 2 system, Kuraray Medical, Tokyo, Japan); various properties of this prototype material have been investigated in detail by many in vitro tests ${ }^{4,10-12)}$. When the experimental primer containing MDPB at $5 \%$ was kept in contact with planktonic Streptococcus mutans $\left(1 \times 10^{8} \mathrm{CFU}\right)$, all bacteria were killed within $30 \mathrm{sec}^{10)}$. The experimental MDPBcontaining primer was also inhibitory against

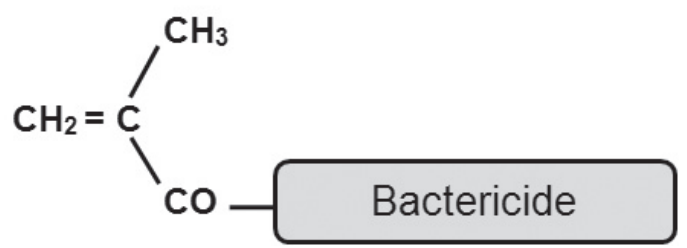

Fig. 1 Antibacterial monomer with a polymerizable group and bactericide in the molecule.

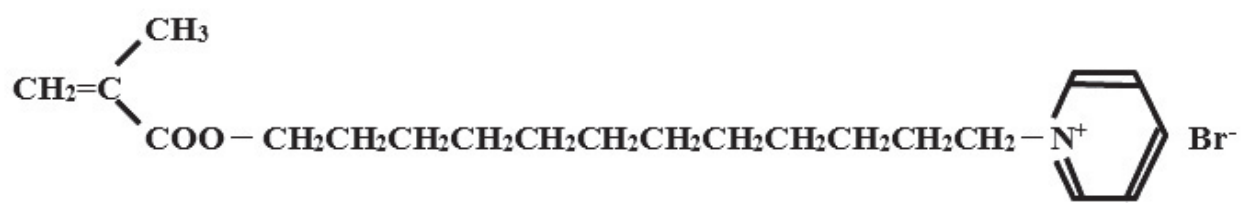

Fig. 2 MDPB synthesized by combining a quaternary ammonium dodecylpyridinium bromide and a methacryloyl group.

Table 1 Minimum bactericidal concentrations of unpolymerized MDPB ( $\mu \mathrm{g} / \mathrm{mL})$ for various caries-associated bacteria

$\begin{array}{lc}\text { Streptococcus mutans } & 62.5-125 \\ \text { Streptococcus sobrinus SL-1 } & 62.5 \\ \text { Streptococcus sanguinis NCTC7863 } & 31.3 \\ \text { Streptococcus salivarius NCTC8618 } & 31.3 \\ \text { Lactobacillus plantarum ATCC14917 } & 15.6 \\ \text { Lactobacillus fermentum ATCC14931 } & 15.6 \\ \text { Propionibacterium acnes ATCC6919 } & 62.5 \\ \text { Bifidobacterium bifidum ATCC29521 } & 62.5 \\ \text { Peptostreptococcus asaccharolyticus ATCC14963 } & 31.3\end{array}$

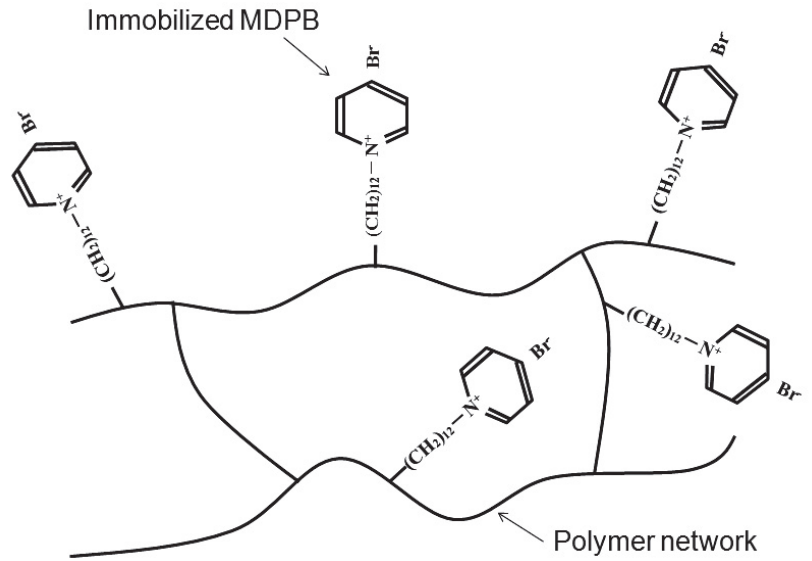

Fig. 3 Immobilization of the antibacterial component by polymerization of MDPB.

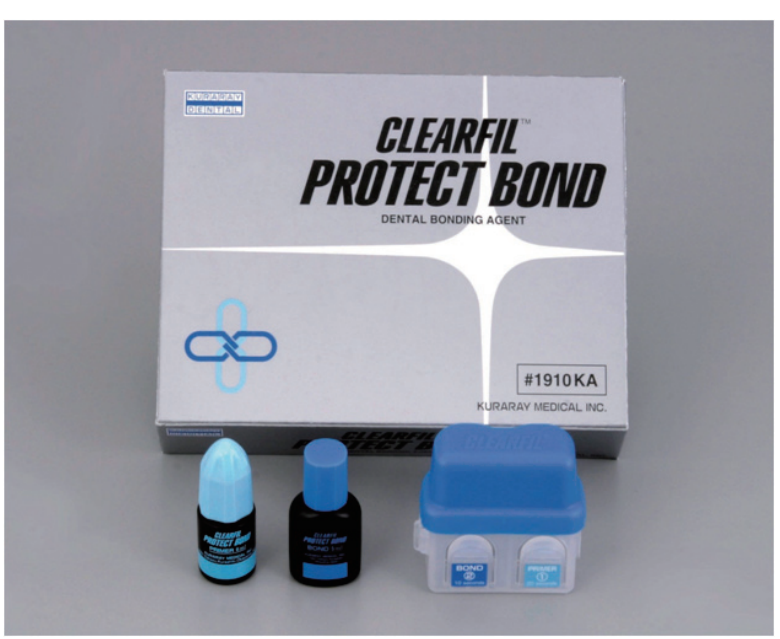

Fig. 4 The world's first antibacterial adhesive system (Clearfil Protect Bond), employing a self-etching primer containing 5\% MDPB. 


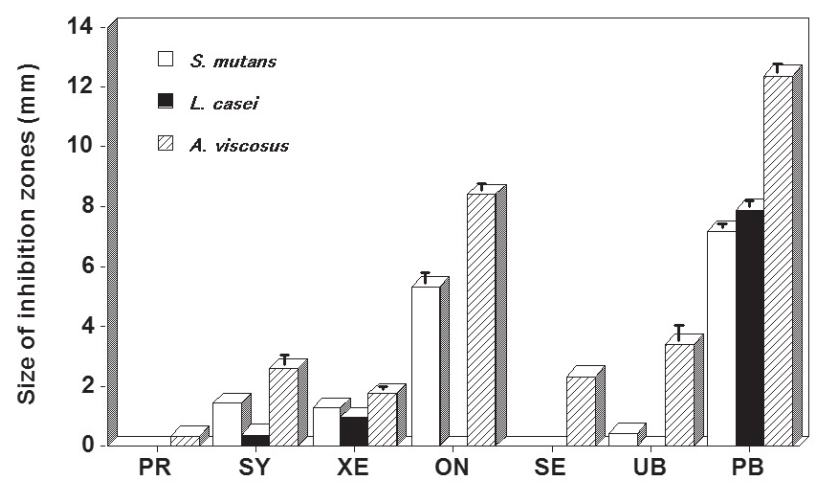

Fig. 5 Inhibitory effects of proprietary self-etching adhesives/primers against caries-associated bacteria: S. mutans, L. casei, and A. viscosus. PR: Prime \& Bond NT, SY: Syntac SC, XE: Xeno CF Bond, ON: One-up Bond F, SE: Clearfil SE Bond primer, UB: Unifil Bond primer, PB: Clearfil Protect Bond primer

Actinomyces, Lactobacillus, and anaerobic bacteria isolated from human carious dentinal lesions ${ }^{4,10)}$.

Based on these findings obtained for the experimental material, MDPB was utilized successfully in the commercial adhesive (Clearfil Protect Bond, Kuraray Medical, Fig. 4). This adhesive is a two-step self-etching system, employing a singlebottled 5\% MDPB-containing primer. Owing to the effects of the incorporated MDPB, the primer of Clearfil Protect Bond system shows cavitydisinfecting effects ${ }^{13)}$. Therefore, this adhesive contributes to a reduction in the risk of secondary caries and to saving the pulp in caries treatment.

Antibacterial activity tests using agar-disc diffusion methods clearly demonstrated the effectiveness of Clearfil Protect Bond primer against cariesrelated bacteria ${ }^{13-15)}$. Among the several proprietary self-etching primers/adhesives examined, Clearfil Protect Bond primer was the only material that could inhibit $S$. mutans, L. casei and A. viscosus effectively (Fig. 5). The self-etching adhesives are more or less acidic, and exhibit antibacterial activity to some extent $^{16-18)}$. However, they are not effective against lactobacilli that can survive even in a low $\mathrm{pH}$ environment. It is noteworthy that Clearfil Protect Bond primer can inhibit the growth of acid-tolerant species such as lactobacilli, due to the intrinsic antibacterial activity of MDPB.

In vitro bactericidal activity tests using artificially infected dentin specimens indicated that Clearfil Protect Bond primer can kill the inhabitant bacteria in demineralized dentin ${ }^{15,19)}$. In this test, a dentinal block was obtained from human tooth crown and a bacterial suspension (S. mutans, L. casei or A. viscosus) was impregnated after demineralizing the block with an acid solution, producing artificially

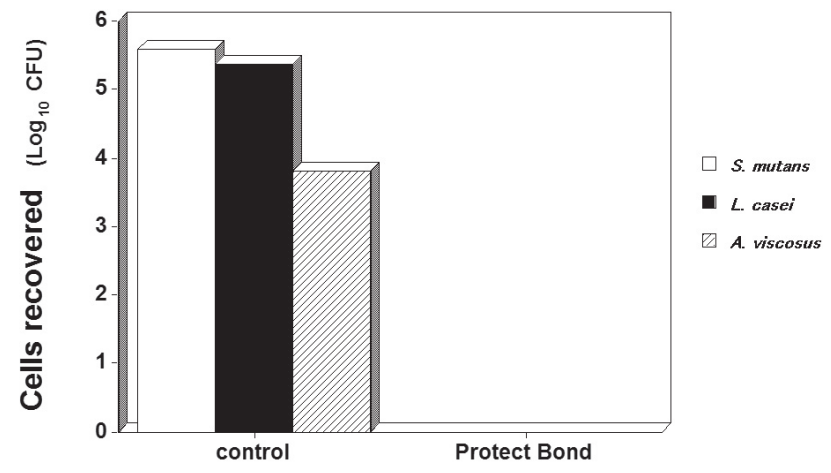

Fig. 6 The number of viable bacteria in the dentin block artificially infected with S. mutans, L. casei, or A. viscosus. Bacteria was completely killed by application of MDPB-containing primer (Clearfil Protect Bond primer) due to the strong bactericidal effects shown by MDPB. Control indicates bacterial number recovered without any treatment.

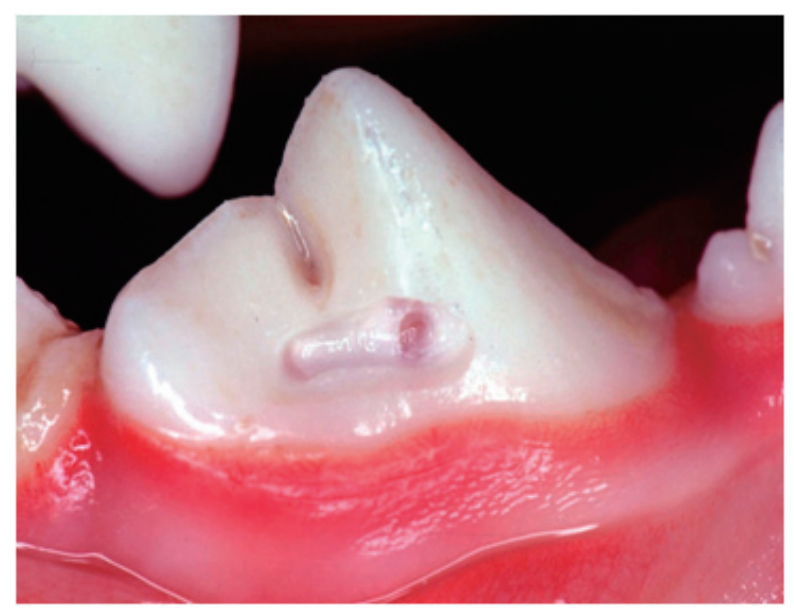

Fig. 7 Infected cavity prepared on a beagle dog tooth.

infected dentin containing a high amount of bacteria. It was confirmed that all bacteria in the artificial caries block could be killed by application of Clearfil Protect Bond primer for $20 \mathrm{sec}$ (Fig. 6). The effectiveness of Clearfil Protect Bond primer was also exemplified by studies using a natural caries lesion of a freshly extracted human tooth ${ }^{20}$. By this test, MDPB was shown to be able to disinfect the heavily infected cavity containing mixed flora.

To evaluate the clinical usefulness of MDPB, an animal model was established and the antibacterial activity of the primer containing MDPB was assessed in more realistic condition ${ }^{21}$. In this model, the cavity was prepared on a sound tooth of a beagle dog and inoculated with $S$. mutans to simulate the residual infection after caries removal (Fig. 7). The bacteria were completely inactivated by the application of the 
experimental primer containing 5\% MDPB (Table 2), indicating that the MDPB-containing self-etching primer could exhibit antibacterial effects not only in vitro but also in vivo.

The fact that such cavity disinfecting effects are beneficial for preserving the pulp was demonstrated by the animal tests using the same model of infected cavity $^{211}$. Composite filling was performed with the antibacterial primer containing 5\% MDPB to the infected cavity, and the pulp was histopathologically evaluated after 7-75 days. While the positive control without application of any material or the restoration using parent primer without MDPB resulted in the occurrence of moderate to mild inflammatory responses of the pulp, even after 75 days, no inflammation was observed for the restoration using the MDPB-containing primer (Fig. 8, Table 3). The other study, which examined the usage of Clearfil Protect Bond to infected non-exposed and exposed cavities, supported the effects of the MDPBcontaining primer to maintain pulp vitality and primary odontoblastic function ${ }^{22}$. Therefore, by the application of antibacterial primer incorporating MDPB to the deep cavity with possible residual infection, bacteria can be eradicated and inflammation of the pulp can be protected.

There are several studies that describe the addition of antibacterial components in adhesive resins ${ }^{18}$. In most cases, soluble antimicrobials were incorporated to show antibacterial activity dependent upon their release. The disadvantage of these approaches is harmful effects on the production and maintenance of integral adhesive interfaces. Deterioration of adhesive interface is inevitable by the release of the agents from cured resin, and this results in low durability of restoration. In addition, disturbance of resin polymerization sometimes occurs by inclusion of the additives, leading to reduction in mechanical properties of the interface and enhancement of interfacial deterioration as ageing. In the case of incorporation of MDPB, the antibacterial component is immobilized at the bonding interface after setting of restorations. The
Table 2 The number of bacteria recovered (CFU) after application of the primers or disinfectant to the infected cavity

\begin{tabular}{lc}
\hline No treatment & $(4.74 \pm 3.98) \times 10^{5}$ \\
Liner Bond 2 primer & $(1.94 \pm 3.35) \times 10^{5}$ \\
$5 \%$ MDPB-containing primer & 0 \\
Cavity disinfectant* & 0 \\
\hline
\end{tabular}

$\mathrm{n}=5,{ }^{*}$ Consepsis (Ultradent) containing $2 \%$ chlorhexidine digluconate
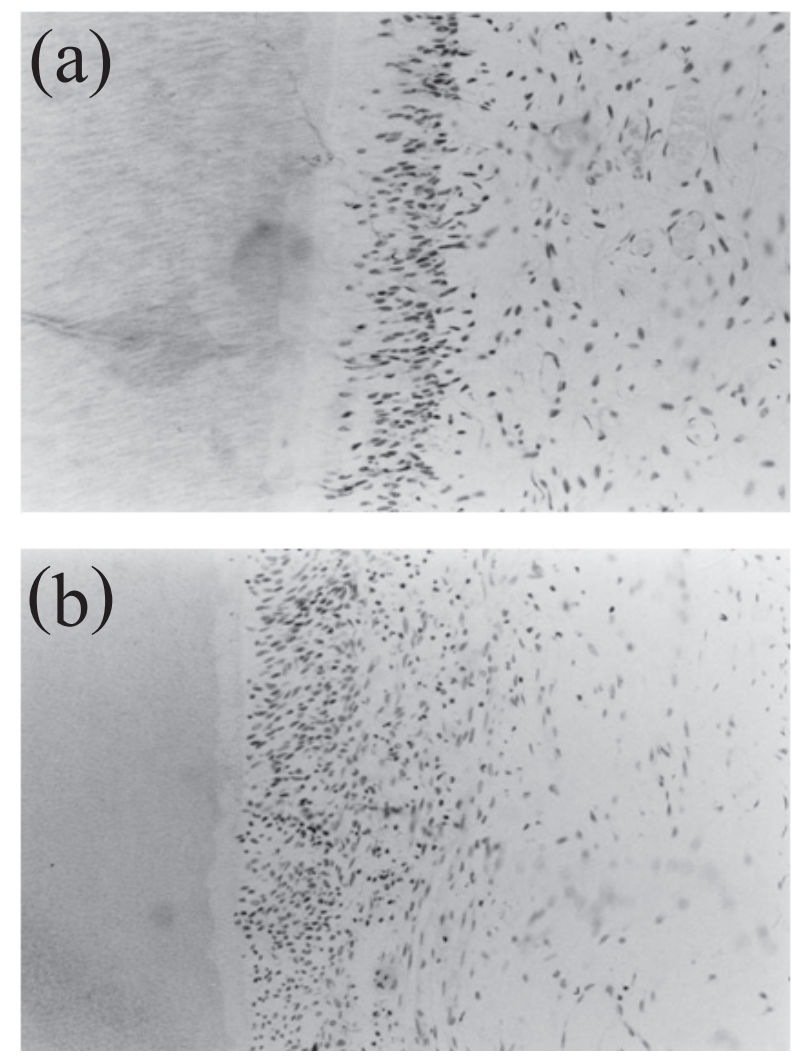

Fig. 8 The 7-day pulp after restoration using 5\% MDPBcontaining primer (a) or without any treatment as the control (b). Note that inflammatory response can be seen for the control, while there is no sign of inflammation for the antibacterial primer group.

Table 3 Pulpal responses after restoration of the infected cavity

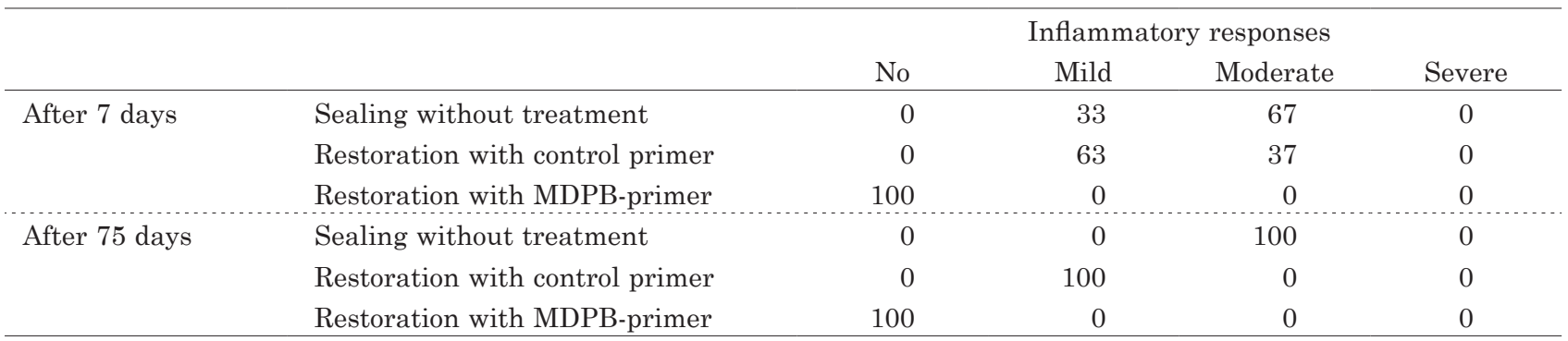

Control primer: Clearfil Liner Bond 2 primer, MDPB-primer: antibacterial primer containing 5\% MDPB. The values represent the percentage to the total specimens. 

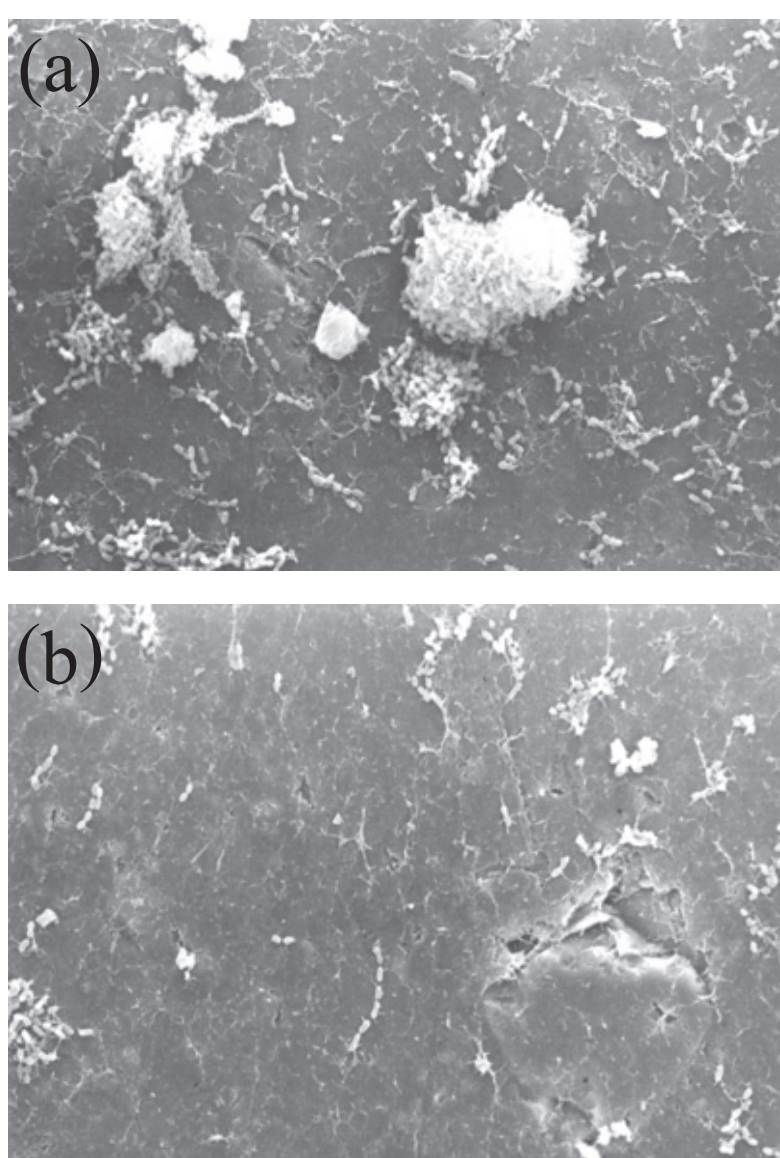

Fig. 9 In vitro plaque accumulation on the control composites (a) and the experimental composites loaded with antibacterial PPRF (b) after $24 \mathrm{~h}$ of incubation in $S$. mutans suspension. Inhibition of maturation of plaque can be clearly seen for the antibacterial composites.

immobilized component of MDPB is not released from the interface, contributing to the durability of restorations. By agar-disc diffusion tests using an intervening dentin disc, Schmalz et al. ${ }^{14)}$ examined the production of inhibitory zones by Clearfil Protect Bond applied onto dentin. They found that no inhibition zones were produced under the dentin disc when the adhesive applied was light-cured, indicating sufficient curing of MDPB in the primer. By in vivo tests, Clearfil Protect Bond proved to produce more durable interface than the conventional adhesives in the oral environment in terms of bond strength to dentin ${ }^{23)}$. No reduction in dentin bond strength for the restorations using Clearfil Protect Bond was demonstrated even after function for 1 year in human mouth. In addition to its excellent bonding ability to sound or caries-affected dentin and enamel ${ }^{24-28)}$, many other in vitro tests also supported the prominent bonding durability of this adhesive ${ }^{29-31}$. These findings appear to reflect the advantages of incorporation of the antibacterial monomer.

\section{Antibacterial filler with immobilized MDPB}

It has been found that cured resins containing MDPB demonstrate an activity to inhibit the growth of bacteria on their surface, thereby acting as a so called "contact inhibitor". This property has been suggested from the results of antibacterial activity tests of MDPB-containing resin matrix for composites and bonding resins $\mathrm{s}^{1,2,8,32)}$.

The idea to utilize the antibacterial monomer MDPB for a prepolymerized resin filler (PPRF) has been tried as the surface with immobilized antibacterial component at high concentrations could be obtained. PPRF containing approximately 18\% MDPB was prepared and its inhibitory effects on bacterial growth were studied using $S$. mutans $^{33,34)}$. Although the release of unpolymerized MDPB from the antibacterial PPRF was less than the minimum inhibitory concentration (MIC) value, the growth of $S$. mutans was inhibited when incubated in contact with the experimental PPRF ${ }^{33)}$. Composites loaded with this experimental PPRF exhibited inhibitory effects on the growth of $S$. mutans as well, and in vitro plaque accumulation on its surface was reduced compared with control composites without MDPB (Fig. 9) ${ }^{34)}$. The advantages of bactericide-immobilized composite are the long-lasting antibacterial effect and no deterioration in mechanical properties after ageing in a wet environment.

However, the antibacterial activities shown by the bactericide-immobilized resins are mainly bacteriostatic, and not as intensive as those shown by the unpolymerized MDPB. What remains to be determined about the effects of immobilized MDPB is elucidation of the mechanism by which it inhibits bacterial activity. Reduction in effects owing to the adsorption of proteins on the surface is a general drawback of contact inhibitor, and this problem needs to be resolved to achieve clinically effective materials.

\section{Caries management by antibacterial resins}

People today tend to be able to preserve their teeth for longer, showing a higher prevalence of gingival recession and exposure of the root surfaces to the oral environment. This condition, in addition to a lower salivary flow rate and poor oral hygiene, increases the risk of root surface caries in elderly people. Epidemiological studies have demonstrated that the prevalence of root surface caries in the elderly population is more than $50 \%^{35,36)}$. Application of antibacterial resins may be one option for the management of root surface caries, especially beneficial for arresting active lesions. This regimen is based upon inactivation of the bacteria within a 
lesion and simultaneous sealing of the surface to deprive any viable bacteria from nutrients and ingress of acids.

Using an artificial caries lesion with extensive demineralization, we found that the primer incorporating 4\% MDPB was able to penetrate into lesions to a depth of more than $150 \mu \mathrm{m}$ and to kill bacteria completely within demineralized dentin ${ }^{37)}$. These results indicated the possible benefit of using an adhesive system employing an MDPB-containing primer and bonding resin to inhibit the progression of root caries. Therefore, artificial caries lesions were prepared using either an acid-gel or an S. mutans culture technique on the roots of extracted human teeth, and the progression of the lesions after application of MDPB-containing adhesive systems was examined. Further demineralization was completely prevented by the MDPB-containing adhesive system, whilst lesions managed with proprietary adhesives showed limited inhibition of the progression of demineralization (Table 4) ${ }^{38}$. Based on these in vitro findings, an evaluation of the clinical usefulness of the management option through a combination of the antimicrobial activity of MDPBcontaining primer and sealing of the demineralized dentin with resins is under investigation.

\section{Antibacterial coating/sealer}

Aiming at the achievement of a new preventive measure for root surface caries, coating of the surface with adhesive systems has been investigated ${ }^{39-41)}$. We previously reported that the application of a multifunctional methacrylate-based surface-coating resin with a dentin primer was effective to seal the surface and subsequently protect roots from demineralization ${ }^{42}$. Addition of antibacterial activity on the surface is considered to provide further benefit for the prevention of caries, and various properties of experimental coating resins incorporating MDPB are being evaluated.

It would be also possible to utilize unfilled resins containing $\mathrm{MDPB}^{43)}$ as root canal sealers in conjunction with disinfection of root canals by an MDPB-containing primer.

Table 4 The mean depth of the lesion ( $\mu \mathrm{m}$ ) produced by S. mutans culture

\begin{tabular}{ll}
\hline 2 w culture (before application of adhesives) & 260 \\
4 w culture without application of adhesive & 417 \\
Single Bond & 353 \\
MDPB-containing primer/bonding resin & 276 \\
\hline
\end{tabular}

The adhesive was applied to the lesion produced by $2 \mathrm{w}$ culture, and incubated for additional $2 \mathrm{w}$.

\section{ANTIBACTERIAL GLASS-IONOMER CEMENTS FOR ART}

\section{In vitro studies}

Atraumatic restorative treatment (ART) is one minimal intervention approach in which demineralized tooth tissues are removed using manual instruments, and the cavity, including adjacent pits and fissures, is restored using a filling material, usually a glass-ionomer cement (GIC). Since ART can be performed under circumstances where neither electricity nor local anesthesia is required, it is possible that insufficient carious tissues are removed in the process of cavity cleaning. Accordingly, improvement of filling materials to overcome the problems caused by incomplete removal of infected dentin will lead to an increase in the success rate of ART.

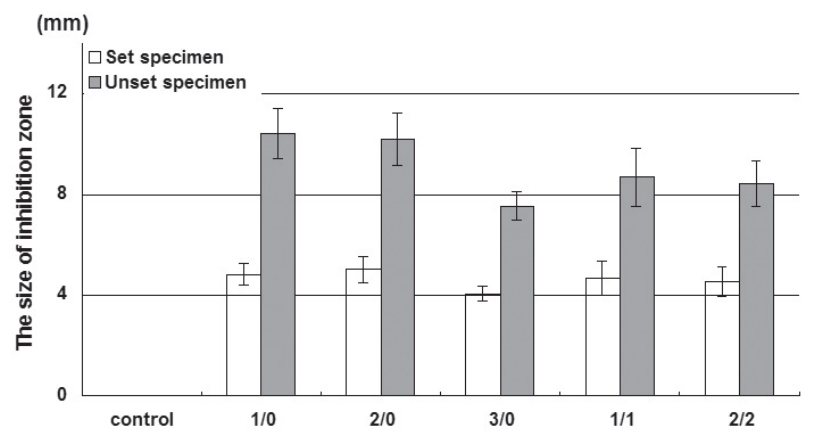

Fig. 10 The size of inhibition zones $(\mathrm{mm})$ produced against S. mutans NCTC10449 by set or unset specimens of CHX-containing GICs. 1/0, 2/0, 3/0, 1/1, and $2 / 2$ indicate the $(\mathrm{w} / \mathrm{w}) \%$ ratio of $\mathrm{CHX}$ diacetate/ CHX dihydrochloride in the powder of control GIC (Fuji IX).

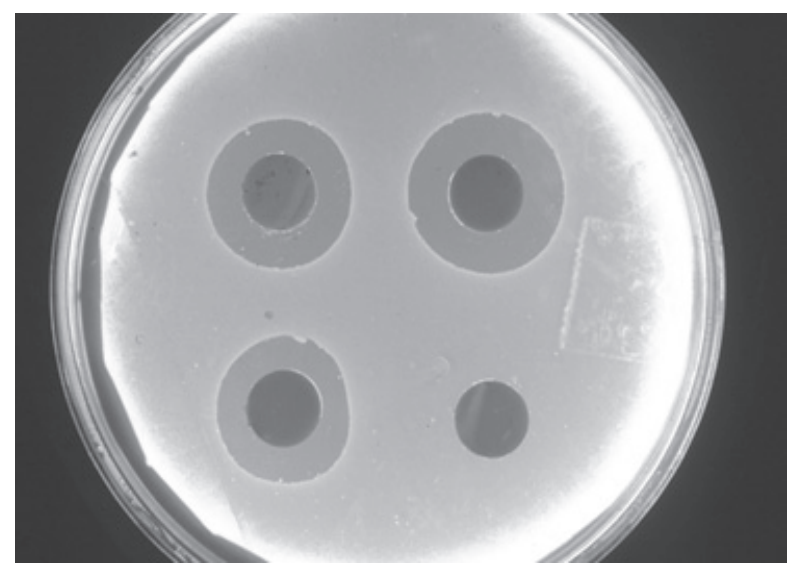

Fig. 11 Inhibition halos produced against $S$. mutans NCTC10449 by set specimens of CHX-containing GICs. Top left: 1/0 specimen, bottom left: $1 / 1$ specimen, top right $2 / 2$ specimen, bottom right: control $(0 / 0)$. 
In the attempts to achieve GIC showing antibacterial effects, we selected cholorhexidine (CHX), which is known to be bactericidal against cariesassociated bacteria, as an antimicrobial to be incorporated. Since the ART approach is indicative for use in posterior teeth, it is critical that the materials show physical properties strong enough to resist an occlusal load. Therefore, to determine the optimal concentration of CHX incorporation, CHX diacetate combined with the less water-soluble compound CHX dihydrochloride was added to the

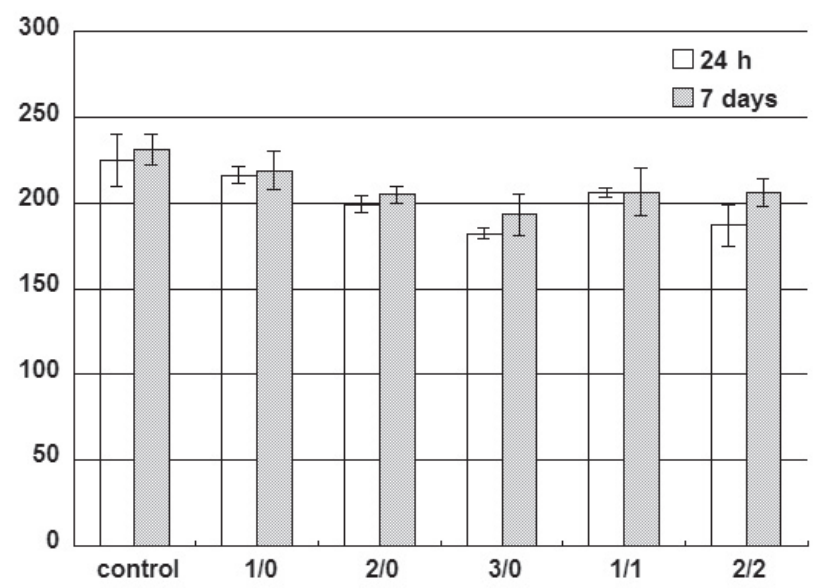

Fig. 12 Compressive strengths (MPa) of CHX-containing GICs after $24 \mathrm{~h}$ or 7 days of immersion in water. $1 / 0,2 / 0,3 / 0,1 / 1$, and $2 / 2$ indicate the $(\mathrm{w} / \mathrm{w}) \%$ ratio of $\mathrm{CHX}$ diacetate/CHX dihydrochloride in the powder of control GIC (Fuji IX). powder of Fuji IX (GC, Tokyo, Japan) to obtain concentration ratios of $1 / 0,2 / 0,3 / 0,1 / 1$, or $2 / 2(w /$ $\mathrm{w}) \%$, and the physical properties and bond strength of these experimental GICs, as well as their antibacterial effects, were evaluated $^{44)}$. By agar-diffusion methods, all experimental GICs showed antibacterial activity against $S$. mutans, L. casei or A. naeslundii due to the release of CHX at both set and unset stages. However, the sizes of the inhibition zones were not dependent upon CHX content (Fig. 10, 11). Analysis using high-

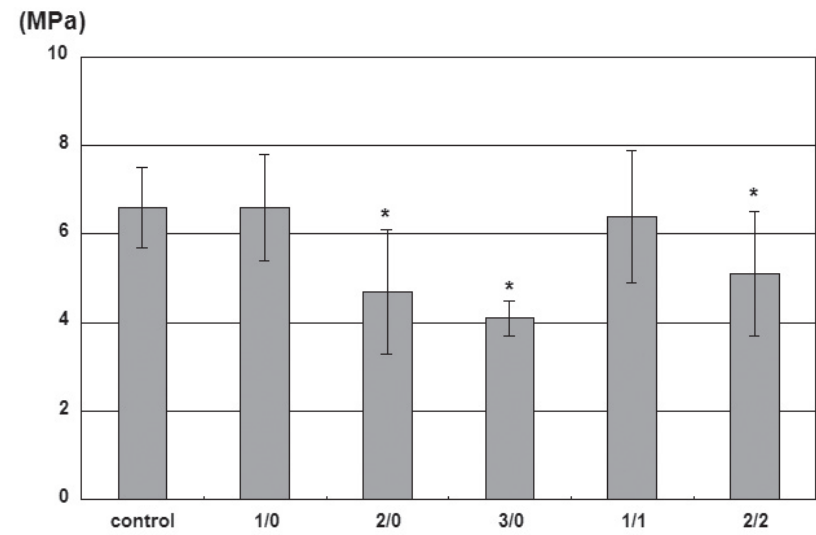

Fig. 13 Tensile bond strengths (MPa) of CHX-containing GICs to bovine dentin. 1/0, 2/0,3/0, 1/1, and $2 / 2$ indicate the $(\mathrm{w} / \mathrm{w}) \%$ ratio of $\mathrm{CHX}$ diacetate/ $\mathrm{CHX}$ dihydrochloride in the powder of control GIC (Fuji IX).

Table 5 The mean number of log-transformed mutans streptococci (MS), lactobacilli (LB) and total bacteria (CFU/mg dentin)

Affected dentin

\begin{tabular}{|c|c|c|c|c|c|c|}
\hline & \multicolumn{2}{|c|}{ MS } & \multicolumn{2}{|c|}{$\mathrm{LB}$} & \multicolumn{2}{|c|}{ total bacteria } \\
\hline & day 0 & day 7 & day 0 & day 7 & day 0 & day 7 \\
\hline \multicolumn{7}{|c|}{ Anaerobic incubation } \\
\hline Control & 1.89 & 1.48 & 2.05 & 1.59 & 2.98 & 2.33 \\
\hline CHX-GIC & 2.11 & 0.78 & 2.05 & 0.88 & 2.92 & 1.35 \\
\hline \multicolumn{7}{|c|}{ Aerobic incubation } \\
\hline Control & 1.22 & 1.27 & 1.92 & 1.59 & 2.69 & 2.04 \\
\hline CHX-GIC & 1.30 & 0.80 & 1.73 & 0.87 & 2.89 & 1.13 \\
\hline
\end{tabular}

Infected dentin

\begin{tabular}{|c|c|c|c|c|c|c|}
\hline & \multicolumn{2}{|c|}{ MS } & \multicolumn{2}{|c|}{ LB } & \multicolumn{2}{|c|}{ total bacteria } \\
\hline & day 0 & day 7 & day 0 & day 7 & day 0 & day 7 \\
\hline \multicolumn{7}{|c|}{ Anaerobic incubation } \\
\hline Control & 3.34 & 1.42 & 3.27 & 1.98 & 4.68 & 2.57 \\
\hline CHX-GIC & 3.40 & 1.09 & 2.83 & 1.36 & 4.26 & 1.92 \\
\hline \multicolumn{7}{|c|}{ Aerobic incubation } \\
\hline Control & 2.02 & 1.17 & 2.75 & 1.82 & 4.25 & 2.57 \\
\hline CHX-GIC & 1.95 & 0.95 & 2.69 & 1.23 & 3.98 & 1.61 \\
\hline
\end{tabular}


performance liquid chromatography revealed that CHX concentrations released from set materials were not different, regardless of CHX content. Incorporation of $\mathrm{CHX}$ diacetate at $2 \%$ or greater significantly decreased compressive strength and adversely affected bond strength to dentin (Fig. 12, 13). Conclusively, we found that incorporation of $1 \%$ CHX diacetate is optimal for clinical use in terms of antibacterial activities, agent-release profile, physical properties and bonding ability to tooth substrates.

\section{In vivo effects}

To test the in vivo antibacterial effects of GIC containing 1\% CHX diacetate, a pilot clinical study was carried out, recruiting 6- to 11-year-old children with occlusal lesions in a molar ${ }^{45)}$. The cavity walls and one half of the floor were cleaned and restored with Fuji IX or the experimental GIC containing 1\% CHX diacetate. Dentine samples were taken from the cleaned (affected dentin) and non-cleaned area (infected dentin) at baseline and 7 days after restoration, and cultivated anaerobically and aerobically to count the number of mutans streptococci, lactobacilli and the total viable bacteria. Although a significant decrease in anaerobic and aerobic bacterial counts was observed for both materials seven days post treatment, fewer bacteria survived in the CHX-containing GIC group compared with the control GIC group (Table 5), indicating the effectiveness of incorporation of CHX. For affected dentin, CHX-containing GIC reduced the total bacteria to approximately $10 \mathrm{CFU}$ in the mean value over the 7 day period, and the number of mutans streptococci and lactobacilli were both reduced to less than $10 \mathrm{CFU}$. Even for infected dentin, total bacterial number was reduced to less than $100 \mathrm{CFU}$ by placement of the CHX-containing GIC, showing greater reduction in surviving bacteria than the control GIC. This pilot clinical study revealed the benefits of a CHX-containing GIC to effectively deal with residual infection for ART. Further follow-up study is ongoing to confirm the significance of the experimental GIC to contribute to a better prognosis.

\section{ACKNOWLEDGEMENTS}

This study was, in part, supported by a Grant-in-Aid for Scientific Research No. 19209060 from the Japan Society for the Promotion of Science.

\section{REFERENCES}

1) Imazato S, Torii $\mathrm{M}$, Tsuchitani $\mathrm{Y}$, McCabe JF, Russell RRB. Incorporation of bacterial inhibitor into resin composite. J Dent Res 1994; 73: 1437-1443.

2) Imazato S, Russell RRB, McCabe JF. Antibacterial activity of MDPB polymer incorporated in dental resin. J Dent 1995; 23: 177-181.

3) Imazato S, Ebi N, Tarumi H, Russell RRB, Kaneko T, Ebisu S. Bactericidal activity and cytotoxicity of antibacterial monomer MDPB. Biomaterials 1999; 20: 899-903.

4) Imazato S, Torii Y, Takatsuka T, Inoue K, Ebi N, Ebisu S. Bactericidal effect of dentin primer containing antibacterial monomer methacryloyloxydodecylpyridinium bromide (MDPB) against bacteria in human carious dentin. J Oral Rehabil 2001; 28: 314-319.

5) Yoshikawa K, Clark DT, Brailsford SR, Beighton D, Watson TF, Imazato S, Momoi Y. The effect of antibacterial monomer MDPB on the growth of organisms associated with root caries. Dent Mater J 2007; 26: 388-392.

6) Imazato S, Ohmori $\mathrm{K}$, Russell RRB, McCabe JF, Momoi Y, Maeda N. Determination of bactericidal activity of antibacterial monomer MDPB by a viability staining method. Dent Mater J 2008; 27: 145-148.

7) Imazato S, McCabe JF. Influence of incorporation of antibacterial monomer on curing behavior of a dental resin composite. J Dent Res 1994; 73: 16411645 .

8) Imazato S, Imai T, Russell RRB, Torii M, Ebisu S. Antibacterial activity of cured dental resin incorporating the antibacterial monomer MDPB and an adhesion-promoting monomer. J Biomed Mater Res 1998; 39: 511-515.

9) Imazato S, Tarumi H, Kato S, Ebisu S. Water sorption and colour stability of composites containing the antibacterial monomer MDPB. J Dent 1999; 27: 279-283.

10) Imazato S, Kinomoto $Y$, Tarumi H, Torii M, Russell RRB, McCabe JF. Incorporation of antibacterial monomer MDPB in dentin primer. J Dent Res 1997; 76: 768-772.

11) Imazato S, Ehara A, Torii M, Ebisu S. Antibacterial activity of dentin primer containing MDPB after curing. J Dent 1998; 26: 267-271.

12) Imazato S, Tarumi H, Ebi N, Ebisu S. Cytotoxic effects of composite restorations employing selfetching primers or experimental antibacterial primers. J Dent 2000; 28: 61-67.

13) Özer F, Karakaya S, Ünlü N, Erganis O, Kav K, Imazato $\mathrm{S}$. Comparison of antibacterial activity of two dentin bonding systems using agar well technique and tooth cavity model. J Dent 2003; 31: 111-116.

14) Schmalz G, Ergücü Z, Hiller KA. Effect of dentin on the antibacterial activity of dentin bonding agents. J Endod 2004; 30: 352-358.

15) Imazato S, Kuramoto A, Takahashi Y, Ebisu S, Peters MC. In vitro antibacterial effects of the dentin primer of Clearfil Protect Bond. Dent Mater 2006; 22: 527-532.

16) Imazato S, Imai T, Ebisu S. Antibacterial activity of proprietary self-etching primers. Am J Dent 1998; 11: 106-108.

17) Imazato S, Kuramoto A, Kaneko T, Ebisu S, Russell RRB. Comparison of antibacterial activity of simplified adhesive systems. Am J Dent 2002; 15: 
356-360.

18) Imazato S. Antibacterial properties of resin composites and dentin bonding systems. Dent Mater 2003; 19: 449-457.

19) Kuramoto A, Imazato S, Ebisu S, Walls AWG, Russell RRB. Bactericidal effect of antibacterial primer impregnated into demineralized dentin. J Dent Res 2001; 80 (special issue): 675 .

20) Smith G, Lumley PJ, Ruettermann S, Imazato S, Smith AJ. Antibacterial effects of a self-etching/ priming system for composite resin restorations. J Dent Res 2003; 82 (special issue B): B-343.

21) Imazato S, Kaneko T, Takahashi Y, Noiri Y, Ebisu $\mathrm{S}$. In vivo antibacterial effects of dentin primer incorporating MDPB. Oper Dent 2004; 29: 369-375.

22) Tziafas D, Koliniotou-Koumpia E, Tziafa C, Papadimitriou S. Effects of a new antibacterial adhesives on the repair capacity of the pulp-dentine complex in infected teeth. Int Endod J 2007; 40: 5866.

23) Donmez N, Belli S, Pashley DH, Tay FR. Ultrastructural correlates of in vivo/in vitro bond degradation in self-etch adhesives. J Dent Res 2005; 84: 355-359.

24) Yoshiyama M, Tay FR, Doi J, Nishitani Y, Yamada T, Itou K, Carvalho RM, Nakajima M, Pashley DH. Bonding of self-etch and total-etch adhesives to carious dentin. J Dent Res 2002; 81: 556-560.

25) De Munck J, Van Meerbeek B, Inoue S, Vargas M, Yoshida Y, Armstrong S, Lambrechts P, Vanherle G. Microtensile bond strengths of one- and two-step self-etch adhesives to bur-cut enamel and dentin. Am J Dent 2003; 16: 414-420.

26) Chan KM, Tay FR, King NM, Imazato S, Pashley DH. Bonding of mild self-etching primers/adhesives to dentin with thick smear layers. Am J Dent 2003; 16: 340-346.

27) Sonoda H, Banerjee A, Sherriff M, Tagami J, Watson TF. An in vitro investigation of microtensile bond strengths of two dentine adhesives to caries-affected dentine. J Dent 2005; 33: 335-342.

28) Shinohara MS, Yamauti M, Inoue G, Nikaido T, Tagami J, Giannini M, De Goes MF. Evaluation of antibacterial and fluoride-releasing adhesive system on dentin - microtensile bond strength and acid-base challenge. Dent Mater J 2006; 25: 545-552.

29) Miguez PA, Pereira MP, Swift EJ Jr. One-year tensile bond strengths of two self-etching primers to bovine enamel. J Esthet Rest Dent 2004; 16: 243248.

30) Ansari ZJ, Sadr A, Moezizadeh M, Aminian R, Ghasemi A, Shimada Y, Tagami J, Ansari SJ, Moayedi S. Effects of one-year storage in water on bond strength of self-etching adhesives to enamel and dentin. Dent Mater J 2008; 27: 266-272.

31) Reis AF, Giannini M, Pereira PN. Effects of a peripheral enamel bond on the long-term effectiveness of dentin bonding agents exposed to water in vitro. J Biomed Mater Res B: Appl Biomater 2008; 85: 10-17.

32) Imazato S, Kinomoto Y, Tarumi H, Ebisu S, Tay FR. Antibacterial activity and bonding characteristics of an adhesive resin containing antibacterial monomer MDPB. Dent Mater 2003; 19: 313-319.

33) Imazato S, Ebi N, Takahashi Y, Kaneko T, Ebisu S, Russell RRB. Antibacterial activity of bactericideimmobilized filler for resin-based restoratives. Biomaterials 2003; 24: 3605-3609.

34) Ebi N, Imazato S, Noiri Y, Ebisu S. Inhibitory effect of resin composite containing bactericide-immobilized filler on plaque accumulation. Dent Mater 2001; 17: 485-491.

35) Galan D, Lynch E. Epidemiology of root caries. Gerodontology 1993; 10: 59-71.

36) Imazato S, Ikebe K, Nokubi T, Ebisu S, Walls AWG. Prevalence of root caries in a selected population of older adults in Japan. J Oral Rehabil 2006; 33: 137143.

37) Imazato S, Walls AWG, Kuramoto A, Ebisu S. Penetration of an antibacterial dentine-bonding system into demineralized human root dentine in vitro. Eur J Oral Sci 2002; 110: 168-174.

38) Kuramoto A, Imazato S, Walls AWG, Ebisu S. Inhibition of progression of root caries by an antibacterial adhesive. J Dent Res 2005; 84: 89-93.

39) Swift EJ, Hammel SA, Perdigao J, Wefel JS. Prevention of root surface caries using a dental adhesive. J Am Dent Assoc 1994; 125: 571-575.

40) Grogono AL, Mayo JA. Prevention of root caries with dentin adhesives. Am J Dent 1994; 7: 89-90.

41) Han P, Schaller HG, Gernhardt C, Hellwig E. Influence of two bonding systems on the demineralization of root surface. Oper Dent 1999; 24: 344-350.

42) Kaneshiro AV, Imazato S, Ebisu S, Tanaka S, Tanaka Y, Sano H. Effects of a self-etching resin coating system to prevent demineralization of root surfaces. Dent Mater 2008; 24: 1420-1427.

43) Imazato S, Tay FR, Kaneshiro AV, Takahashi Y, Ebisu S. An in vivo evaluation of bonding ability of comprehensive antibacterial adhesive system incorporating MDPB. Dent Mater 2007; 23: 170-176.

44) Takahashi $\mathrm{Y}$, Imazato S, Kaneshiro AV, Ebisu S, Tay FR, Frencken JE. Antibacterial effects and physical properties of glass-ionomer cements containing chlorhexidine for the ART approach. Dent Mater 2006; 22: 647-652.

45) Frencken JE, Imazato S, Toi C, Mulder J, Mickenautsch S, Takahashi Y, Ebisu S. Antibacterial effect of chlorhexidine containing glass-ionomer cement in vivo; a pilot study. Caries Res 2007; 41: 102-107. 